\title{
The Relationship between Quality of Life, Bone Pain, Skin Pruritus and Depression among Patients with Secondary Hyperparathyroidism undergoing Parathyroidectomy: A Cross-Sectional Study
}

\section{Ting Bi}

Northern Theater General Hospital

Xiaodong Feng ( $\triangle 981613149 @ q q . c o m$ )

Northern Theater General Hospital https://orcid.org/0000-0002-2669-7112

Wei Zhang

Northern Theater General Hospital

Guangming Cheng

Northern Theater General Hospital

Chunhui Wang

Northern Theater General Hospital

Yufu Tang

Northern Theater General Hospital

Sijia Bai

Northern Theater General Hospital

Shuai Guo

Northern Theater General Hospital

Research

Keywords: SHPT, Quality of Life, Bone pain, Skin pruritus, Depression

Posted Date: September 23rd, 2020

DOl: https://doi.org/10.21203/rs.3.rs-26542/v3

License: (c) (1) This work is licensed under a Creative Commons Attribution 4.0 International License. Read Full License 


\section{Abstract}

Background: Secondary hyperparathyroidism (SHPT), a manifestation of chronic kidney disease-mineral bone disorder (CKD-MBD), is common in CKD patients and has significant morbidity and mortality. Quality of life (QOL) in SHPT patients is seriously affected by symptoms such as bone pain and skin pruritus. However, studies have focused on operative effects and hypocalcemia rather than the relationships between QOL, bone pain, skin pruritus and depression symptoms.

Methods: A cross-sectional survey was conducted from January 2017 to December 2019 in a third-class hospital in China. The brief table of the QOL measurement scale (QOL-BREF), a self-designed bone pain and skin pruritus scale and the Self-rating Depression Scale (SDS) were used to estimate QOL, bone pain and skin pruritus, and depression, respectively. Pearson's correlation, multiple linear regression analysis and structural equation modeling (SEM) were used for analysis.

Results: Overall, 320 questionnaires were considered valid (98.46\% effective response rate). The prevalence of bone pain, skin pruritus and depression was $94.06 \%, 69.06 \%$, and $77.81 \%$, respectively. Bone pain, skin pruritus and depression were significantly associated with QOL. In SEM, QOL negatively correlated with bone pain ( $r=-0.509)$, skin pruritus $(r=-0.517)$ and SDS $(r=-0.465)$. Bone pain significantly $(P<0.01)$ and positively correlated with skin pruritus $(r=0.568)$ and SDS $(r=0.450)$. Skin pruritus significantly $(P<0.01)$ and positively correlated with SDS $(r=0.426)$. In addition, QOL partially mediated the association between bone pain or skin pruritus and depression (mediation proportion of $48.62 \%$ or $45.08 \%)$.

Conclusions: Patients with SHPT undergoing Parathyroidectomy(PTX) have high depression levels and poor QOL due to bone pain and skin pruritus. The effects on depression may be fully mediated by the impact of bone pain or skin pruritus on QOL. Thus, bone pain or skin pruritus could increase depression via the intermediary role of QOL.For clinical front-line medical staff,it can relieve bone pain and skin pruritus through timely operation and psychological intervention, so as to improve patients' quality of life and reduce anxiety.At the same time, we should also pay attention to the high-risk groups.

\section{Background}

SHPT is a common complication of CKD that is characterized by derangements in the homeostasis of calcium, phosphorus and vitamin D[1]. SHPT causes high-turnover bone disease, leading to a decrease in bone mass[2]. Loss of bone mass is mainly manifested by increased cortical bone, with a reduction of bone resorption and mineralized bone on the surface of the cortex due to mineralization defects, with severe bone pain and fracture in severe cases[3]. Pruritus is an unpleasant feeling associated with the urge to scratch; it is a subjective symptom that is caused by many factors and has multiple dimensions[4]. The causes of skin pruritus in patients with SHPT may be related to the increase in parathyroid hormone (PTH), hyperphosphatemia and the accumulation of some medium and large molecular toxins, such as $\beta 2-\mathrm{mg}$, in vivo[5]. Parathyroidectomy (PTX) is the main treatment at present 
and might also reduce calcium-phosphorus product concentrations, PTH and inflammatory factors, thus controlling vascular calcification and relieving bone pain and skin pruritus[6].

Studies have shown that low QOL and severe physical discomfort can affect patients' mental health, especially depressive symptoms[7]. In this study, we hypothesize that there is a mediating effect between bone pain, skin pruritus and depression.

QOL is a health concept that comprehensively evaluates the qualitative and quantitative factors such as physical function,psychological,social,economic and emotional factors in a patient's life and is used to evaluate the quality of life in a comprehensive way, which should be based on living standards, but its meaning is more complex and extensive[8]. Kim et al.'s research[9] showed that QOL had a strong correlation with pain and depression or anxiety. McIntyre et al.'s research[10] showed that QOL had a mediating effect between symptoms and depression, which indicated that QOL can indirectly affect depression as a mediating variable. JungHye et al.'s research[11] proposed a QOL protection model, and the model considered QOL as a protective factor that can regulate the negative impact of risk factors on development outcomes.There are many influencing factors of SDS.At present,the current research mainly focuses on the direct effects of pain and QOL on the occurrence of SDS,but the joint effect of pain and QOLon SDS is not explained.In view of this,this study hypothesize that bone pain/skin pruritus can not only directly affect SDS of SHPT patients,but also indirectly affect SDS by changing the intermediary link of QOL.

In this study, it is assumed that QOL plays a regulatory role in the mediation model of bone pain, skin pruritus and depression.

In summary, this study hypothesizes that there is a relationship between QOL, bone pain, skin pruritus and depression and that QOL has a mediating effect between bone pain, skin pruritus and depression.

\section{Methods}

\section{Participants and data collection}

A cross-sectional study was conducted in a third-class hospital in Liaoning Province, China, from January 2017 to December 2019. The patients with SHPT undergoing PTX were aware of the content of the survey before their participation, and on the day before the questionnaires were distributed, with the agreement of the Northern Theater General Hospital ethics committee, we issued informed consent forms to each patient. In this study,the inclusion criteria was all patients needed PTX and signed informed consent and the exclusion criteria was severe cardiac insufficiency and cognitive dysfunction.A total of 325 questionnaires were distributed, and those that were missing more than $20 \%$ of values or had low writing quality were excluded from this survey. In total, 320 questionnaires were considered valid (98.46\% effective response rate).

\section{Measures}




\section{Measurement of QOL}

We measured QOL by using the brief table of the quality of life measurement scale (WHOQOL-BREF). The WHOQOL-BREF, a self-reported scale, has 24 items, and each item has a 5-point Likert-type scale ranging from 1 to 5 . The total score of the scale ranges from 16 to 80 , and higher scores indicate better QOL. There are four dimensions in this instrument: the physiological dimension, the psychological dimension, the social relation dimension and the environmental dimension[12]. In this study, the Cronbach's a coefficient of internal consistency for the overall scale was 0.857 .

\section{Measurement of bone pain and skin pruritus}

We used the Visual Analog Scale (VAS) to measure bone pain. This scale draws a long horizontal line on the paper; one end of the horizontal line is 0 , indicating no pain, the other end is 10 , indicating severe pain, and the middle region indicates different degrees of bone pain[13]. This method was also used to measure the degree of skin pruritus.

\section{Measurement of depression}

The Self-rating Depression Scale (SDS) is composed of 20 items and 4 dimensions: psychoemotional symptoms, somatic disorders, neuromotor disorders and psychological disorders. The SDS asks about the degree of depression in patients using a 4-point Likert Scale that ranges from never (1 point) to always (4 points), and the total score ranges from 20 to 80[14]. In this study, the Cronbach's a coefficient of internal consistency for the overall scale was 0.842 .

\section{Statistical analysis}

SPSS 21.0 and AMOS 23.0 were used for the statistical analyses. First,single sample K-S test was used to test whether the measurement data conformed to normal distribution. If it was, it was expressed as ' $x \pm s$ ,otherwise it was expressed as median or interquartile range. We conducted descriptive analyses of the sociodemographic variables and Pearson correlation analysis of the other variables. Multiple linear regression analysis was used to test the mediating effect, and the bootstrap method in the AMOS structural equation model was used to further verify the mediating effect. The process of parameter estimations could exclude measurement error[15]. According to related research and theories, a hypothetical relationship model was created and is shown in Figures 1 and 2. Two SEM models were presented, as follows: QOL is an intermediary variable between bone pain, skin pruritus and depression. The structural path hypothesis is that bone pain and skin pruritus have direct and indirect effects through depression. Furthermore, it shows the results for goodness-of-fit indexes obtained with SEM for the total sample and individual samples.

\section{Results}

\section{Demographic characteristics of the participants}


There were 320 patients with SHPT undergoing PTX in this study: 172 (53.8\%) were males, and 148 (46.2\%) were females. 295(92.19\%) had different degrees of forgetfulness,302(94.38\%) had varying degrees of fatigue.The age of the patients ranged from 20 to 70 years. The years of renal failure ranged from 2 to 30 , and the average was $10.71 \pm 4.64$ years. The years of SHPT ranged from 1 to 9 , and the average was $3.07 \pm 2.13$ years.

\section{Descriptive statistics}

In this study,the measurement data showed a normal distribution,so it was expressed as ' $\mathrm{x} \pm \mathrm{s}$. The QOL score ranged from 25 to 68 , with an average of $41.76 \pm 8.15$. The scores for bone pain and skin pruritus ranged from 1 to 10 , with averages of $5.08 \pm 2.65$ and $3.58 \pm 3.19$, respectively. The years of bone pain ranged from 0 to 10 , and the average was $2.30 \pm 1.95$. The years of skin pruritus ranged from 0 to 8 , and the average was $2.13 \pm 2.05$. The SDS score ranged from 20 to 65 , and the average was $46.04 \pm 8.98$. The preoperative PTH ranged from 588 to $2934 \mathrm{pg} / \mathrm{ml}$, and the average was $1729.15 \pm 376.20 \mathrm{pg} / \mathrm{ml}$. The serum calcium ranged from 1.95 to $2.93 \mathrm{mmol} / \mathrm{L}$, and the average was $2.43 \pm 0.19 \mathrm{mmol} / \mathrm{L}$. The serum phosphorus ranged from 1.30 to $3.59 \mathrm{mmol} / \mathrm{L}$, and the average was $2.38 \pm 0.52 \mathrm{mmol} / \mathrm{L}$. The comparative results of each scale for patients with SHPT undergoing PTX with different demographic characteristics are shown in Table 1.

\section{Preliminary correlation analyses}

First,the correlation analysis of renal replacement therapy (RRT) type,renal failure time,dialysis vintage,duration of SHPT and QOL,bone pain,skin pruritus,SDS showed that renal failure time was significantly $(\mathrm{P}<0.01)$ negatively correlated with QOL $(\mathrm{r}=-0.232)$ and positively correlated with skin pruritus $(r=0.142)$ and SDS $(r=0.149)$, other variables were not relevant. The Pearson correlation analysis of QOL, bone pain, skin pruritus and SDS is presented in Table 2. The results showed that QOL was significantly $(P<0.01)$ and negatively correlated with bone pain $(r=-0.509)$, skin pruritus $(r=-0.517)$ and SDS $(r=-0.465)$. Bone pain was significantly $(P<0.01)$ and positively correlated with skin pruritus $(r=0.568)$ and SDS $(r=0.450)$. Skin pruritus was significantly $(P<0.01)$ and positively correlated with SDS $(r=0.426)$.

\section{Testing for the mediation effect}

The variable was normalized first, and three regression equations were established according to the purpose of this study. Equation 1: depression as the dependent variable and bone pain or skin pruritus as the independent variable; Equation 2: QOL as the dependent variable, with SDS and bone pain or skin pruritus as the independent variable; and Equation 3: SDS as the dependent variable, with QOL and bone pain or skin pruritus as the independent variable. The results showed that bone pain and skin pruritus could significantly predict depression $(\beta=0.485 / 0.375, P=0.000)$ and that bone pain and skin pruritus could significantly predict QOL $(\beta=-0.407 /-0.337, P=0.000)$. When bone pain or skin pruritus and QOL predicted SDS at the same time, the predictions were significant $(\beta=0.310 / 0.223, P=0.000$; $\beta=-0.429 /-0.450, P=0.000$ ). This indicated that the direct or indirect effects of bone pain or skin pruritus 
on SDS were significant; that is, QOL plays an intermediary role between bone pain or skin pruritus and SDS. The results are shown in Tables 3 and 4.

\section{Verification of the mediating effect}

On the basis of multivariate regression analysis, the bootstrap method was used to further test the intermediary effect of QOL. To test the intermediary effect, we needed to establish a structural equation model, and we used AMOS 23.0 to test it. The specific path analysis diagram is shown in Figures 1 and 2.

The structural equation model fit results showed that CMIN/DF=2.437/1.202 (<3); AGFI=0.928/0.965, $\mathrm{GFI}=0.960 / 0.980, \mathrm{TLI}=0.961 / 0.994, \mathrm{IFI}=0.973 / 0.996$, and $\mathrm{CFI}=0.973 / 0.996(>0.9)$; and SRMR $=0.042 / 0.026$ and RMSEA $=0.067 / 0.025(<0.08)$, indicating that the model fit well. The results showed that the confidence intervals for both the direct and indirect effects of bone pain and skin pruritus on SDS did not reach 0 . The mediating effect model of QOL was established, and the mediating effect was $0.212 / 0.275$, accounting for $48.62 \% / 45.08 \%$ of the total effect. See Tables 5 and 6 for details.

\section{Discussion}

\section{The depression status of SHPT patients}

Previous studies have shown that Cinacalet can inhibit PTH in a short time[16],but most patients can not afford it because of its high price.Meanwhile,previous studies in our department have shown that patients taking Cinacalcet for more than 5 years can not continue to suppress PTH,but PTX can better suppress PTH,improve symptoms and quality of life.With the transformation of medical models into biological/psychological/social models, the phenomenon of bodily disease accompanied by anxiety and depression has attracted wide attention in the field of medical psychology. The main symptoms of patients with SHPT undergoing PTX are depression, memory loss, insomnia, nightmares, etc[17]. However, there are few investigations on QOL and depression psychology at home and abroad. In this study, patients had a high level of depression, with an incidence of $77.81 \%$. The average SDS score was $46.04 \pm 8.98$, which was higher than the norm[18]. This result indicated that the mental health level of patients with SHPT undergoing PTX was low, which suggested that more attention should be paid to patients with SHPT undergoing PTX in China. The generation of depressive symptoms includes many factors, such as pain, pruritus, occupation, education, income, and insurance[19], but in the early stage of depression, relieving discomfort over time and giving timely social or emotional support can largely avoid the occurrence of depression[20]. The SDS score of male patients in this study was higher than that of female patients; however, this is different from Liu's findings[21], which may be related to the fact that men are under more economic pressure and most patients are unable to work due to hemodialysis. There were significant differences in SDS scores among patients with different occupations, education, incomes, and insurance, which may be because the patients with high education and a good job generally enjoy higher medical insurance reimbursement and take Cinacalet, lanthanum carbonate and other drugs to alleviate SHPT[22]. 


\section{The status of SHPT patients' QOL, bone pain, and skin pruritus}

The vast majority of patients with SHPT undergoing PTX have skin pruritus,bone pain,electrolyte disorder,coupled with long-term dialysis leading to the destruction of various systems of body,which complicates the screening,such as sleep disturbances,fatigue,loss of energy and poor appetite. However,no related surveys on QOL, bone pain, or skin pruritus in patients with SHPT have been reviewed at home or abroad. In this study, the average QOL score was $41.76 \pm 8.15$, which was lower than the norm[23], and the average score for bone pain was 5.08 \pm 2.65 , which was higher than the norm[24]. This was consistent with Galvez-Sanchez's[25] and Rehman's[26] findings. In this study, skin pruritus had a greater impact on the patient's body than bone pain. PTX can improve the symptoms of bone pain caused by bone deficiency and hypocalcemia.

\section{The mediating effect of QOL}

The results of Pearson correlation analysis showed that QOL, bone pain, skin pruritus and depression were significantly correlated with each other and that bone pain and skin pruritus positively predicted the level of depression. This was consistent with the results obtained by Brophy et al[27]. and laid the foundation for the subsequent analysis of mediating effects. Notably, regression analysis revealed that there was a partial mediating effect between bone pain, skin pruritus and depression, which provided a basis for the verification of mediating effects. At the same time, some studies also showed that patients with low QOL have a significantly higher risk of depression, and QOL is an important predictor of depression in patients with SHPT undergoing PTX, which is basically consistent with the results of this study.

The bootstrap method was used to further verify the mediation effect. The results showed that a mediating effect of QOL between bone pain, skin pruritus and depression was established, and the mediating effect was $48.62 \% / 45.08 \%$. BI also confirmed that QOL played a role in mediating depression[28]. In clinical work, we should encourage patients with SHPT undergoing PTX to improve symptoms causing discomfort through PTX in a timely manner, which can greatly reduce depression and improve QOL.

Medical staff should guide family members to actively participate in all aspects of patients' life and treatment,timely grasp the psychological function of patients, and strengthen the care for patients. Encouraging patients to participate in more social activities can buffer the negative effects of various pressures on body and mind,improve sleep quality,reduce depression and other psychological disorders. Therefore,we set up a wechat group for patients with SHPT undergoing PTX to answer the medical problems after discharge online,such as adjusting blood calcium,regular follow-up within the group.The department should carry out the corresponding mental health survey regularly and give timely intervention to the patients with severe pain,pruritus,sleep disorder and depression tendency,and take positive and effective measures to improve mental health level in time. 


\section{Conclusions}

In summary, QOL, bone pain,skin pruritus and depression are closely related in patients with SHPT undergoing PTX and there may be a full mediating effect of QOL between bone pain or skin pruritus and depression. It is suggested that alleviating bone pain and skin pruritus in patients with SHPT undergoing PTX can alleviate the state of depression through the mediating effect of QOL.

\section{Declarations}

\section{Ethics approval and consent to participate}

Not applicable.

\section{Consent for publication}

Not applicable.

\section{Availability of data and materials}

Not applicable.

\section{Competing interests}

The authors declare that they have no competing interests

\section{Funding}

This study was supported by the Foundation for Guidance plan of Liaoning Natural Science.

\section{Authors' Contributions}

Ting Bi carried out the studies, participated in collecting data, and drafted the manuscript. Xiaodong Feng, Wei Zhang, Guangming Cheng, and Chunhui Wang performed the statistical analysis and participated in its design. Yufu Tang, Sijia Bai, and Shuai Guo participated in acquisition, analysis, or interpretation of data and draft the manuscript. All authors read and approved the final manuscript.

\section{Acknowledgments}

Thanks to all of the study participants.

\section{List Of Abbreviations}




\begin{tabular}{|ll|}
\hline QOL-BREF & QOL measurement scale \\
\hline SDS & Self-rating Depression Scale \\
\hline SEM & structural equation modeling \\
\hline PTH & parathyroid hormone \\
\hline PTX & Parathyroidectomy \\
\hline QOL-BREF & the quality of life measurement scale \\
\hline VAS & the Visual Analog Scale \\
\hline
\end{tabular}

\section{References}

1. Rottembourg J, Urena-Torres $\mathrm{P}$, Toledano $\mathrm{D}$,et al.: Factors associated with parathyroid hormone control in haemodialysis patients with secondary hyperparathyroidism treated with cinacalcet in realworld clinical practice: Mimosa study. Clin Kidney J. 2019; 12:871-9.

2. Queiroz SM, Andrade A, Oliveira PT, et al.: Correlation of Radiomorphometric Indices of the Mandible and Biochemical Parameters in Patients with Secondary Hyperparathyroidism Due to Chronic Kidney Disease. Eur J Dent. 2019; 13:303-9.

3. Ge Y, Yang G, Wang N,et al.: Bone metabolism markers and hungry bone syndrome after parathyroidectomy in dialysis patients with secondary hyperparathyroidism. INT UROL NEPHROL. 2019; 51:1443-9.

4. Yosipovitch G, Bernhard JD: Clinical practice. Chronic pruritus. N Engl J Med. 2013; 368:1625-34.

5. Shen $\mathrm{Q}, \mathrm{Xiang} \mathrm{W}, \mathrm{Ye} \mathrm{S}$, et al.: Plasma metabolite biomarkers related to secondary hyperparathyroidism and parathyroid hormone. J CELL BIOCHEM. 2019; 120:15766-75.

6. Bellorin-Font E, Vasquez-Rios G, Martin KJ: Controversies in the Management of Secondary Hyperparathyroidism in Chronic Kidney Disease. CURR OSTEOPOROS REP. 2019; 17:333-42.

7. Galvez-Sanchez CM, Montoro Cl, Duschek S, Reyes DPG: Depression and trait-anxiety mediate the influence of clinical pain on health-related quality of life in fibromyalgia. J Affect Disord. 2020; 265:486-95.

8. Um-E-Kalsoom: Gender role in anxiety, depression and quality of life in chronic kidney disease patients. PAK J MED SCI. 2020; 36:251-4.

9. Kim DH, Park JI, Lee JP, et al:: The effects of vascular access types on the survival and quality of life and depression in the incident hemodialysis patients. Ren Fail. 2020; 42:30-9.

10. Mclntyre E, Lauche R, Frawley J, Sibbritt D, Reddy P, Adams J: Physical activity and depression symptoms in women with chronic illness and the mediating role of health-related quality of life. $J$ Affect Disord. 2019; 252:294-9.

11. Jung HM, Kim HY: A health-related quality of life model for patients undergoing haemodialysis. J CLIN NURS. 2020; 29:613-25. 
12. Young Carolyn A,Mills Roger,Al-Chalabi Ammar et alهMeasuring quality of life in ALS/MND: validation of the WHOQOL-BREF.[J] .Amyotroph Lateral Scler Frontotemporal Degener, 2020, 6: 1-9.

13. Escalona-Marfil C, Coda A, Ruiz-Moreno J, Riu-Gispert LM, Girones X: Validation of an Electronic Visual Analog Scale mHealth Tool for Acute Pain Assessment: Prospective Cross-Sectional Study. J MED INTERNET RES. 2020; 22:e13468.

14. Duan Q, Sheng L: Clinical validity of anxiety and depression self rating scale. Chinese Journal of mental health. 2012; 26:676-9.

15. Huang PH: Asymptotics of AIC, BIC, and RMSEA for Model Selection in Structural Equation Modeling. PSYCHOMETRIKA. 2017; 82:407-26.

16. 16.Filipozzi Pierre,Ayav Carole,Erpelding Marie-Line et al. Influence on quality of life from an early cinacalcet prescription for secondary hyperparathyroidism in dialysis.[J] .Pharmacoepidemiol Drug Saf, 2015, 24(2): 187-96.

17. Kim WW, Rhee Y, Kim BS, et al.: Clinical outcomes of parathyroidectomy versus cinacalcet in the clinical management of secondary hyperparathyroidism. ENDOCR J. 2019; 66:881-9.

18. Zhang J, Wu Z, Fang G: Establishment of the national urban norm of the depression scale of the center for flow regulation. Chinese Journal of mental health. 2010; 24:139-43.

19. Ho RC, Mak KK, Chua AN, Ho CS, Mak A: The effect of severity of depressive disorder on economic burden in a university hospital in Singapore. Expert Rev Pharmacoecon Outcomes Res. 2013; 13:54959.

20. Marroquin B: Interpersonal emotion regulation as a mechanism of social support in depression. CLIN PSYCHOL REV. 2011; 31:1276-90.

21. Liu CP, Li XM, Chen HW,et al:: Depression, anxiety and influencing factors in patients with acute pulmonary embolism. Chin Med J (Engl). 2011; 124:2438-42.

22. Cunningham J, Block GA, Chertow GM,et al:: Etelcalcetide Is Effective at All Levels of Severity of Secondary Hyperparathyroidism in Hemodialysis Patients. Kidney Int Rep. 2019; 4:987-94.

23. Liegl G, Petersen MA, Groenvold M,et al.: Establishing the European Norm for the health-related quality of life domains of the computer-adaptive test EORTC CAT Core. EUR J CANCER. 2019; 107:133-41.

24. Mazokopakis EE: Chronic stress and depression are associated with chronic bone pain in Biblical times. SPINE J. 2019; 19:961.

25. Galvez-Sanchez CM, Montoro Cl, Duschek S, Reyes DPG: Depression and trait-anxiety mediate the influence of clinical pain on health-related quality of life in fibromyalgia. J Affect Disord. 2020; 265:486-95.

26. Rehman IU, Lai PS, Kun LS, Lee LH, Chan KG, Khan TM: Chronic Kidney Disease-Associated Pruritus and Quality of Life in Malaysian Patients Undergoing Hemodialysis. THER APHER DIAL. 2020; 24:1725 . 
27. Brophy K, Brahler E, Hinz A, Schmidt S, Korner A: The role of self-compassion in the relationship between attachment, depression, and quality of life. J Affect Disord. 2020; 260:45-52.

28. $\mathrm{Bi}$ T, Zhang $\mathrm{H}$ : Mediating effect of quality of life on pain and anxiety in middle-aged and elderly glaucoma patients. China public health. 2017; 33:509-12.

\section{Tables}

Table 1 Descriptive statistics $(\mathrm{N}=320)$

\begin{tabular}{|lllllllll|}
\hline Variable & QOL & \multicolumn{3}{c}{ Bone Pain } & \multicolumn{2}{l}{ Skin Pruritus } & \multicolumn{2}{l|}{ SDS } \\
\cline { 2 - 9 } & $\mathrm{t} / \mathrm{F}$ & $\mathrm{P}$ & $\mathrm{t} / \mathrm{F}$ & $\mathrm{P}$ & $\mathrm{t} / \mathrm{F}$ & $\mathrm{P}$ & $\mathrm{t} / \mathrm{F}$ & $\mathrm{P}$ \\
\hline Gender & 2.066 & 0.040 & -3.313 & 0.001 & -3.300 & 0.001 & -3.156 & 0.002 \\
\hline Age & 1.635 & 0.181 & 2.325 & 0.075 & 0.428 & 0.733 & 2.524 & 0.058 \\
\hline Marital status & 5.783 & 0.001 & 5.407 & 0.001 & 5.786 & 0.001 & 0.309 & 0.819 \\
\hline Occupation & 2.339 & 0.042 & 4.708 & 0.000 & 4.974 & 0.000 & 10.076 & 0.000 \\
\hline Education & 0.688 & 0.601 & 2.589 & 0.037 & 2.807 & 0.026 & 2.949 & 0.020 \\
\hline Income & 2.185 & 0.090 & 1.453 & 0.228 & 2.153 & 0.093 & 5.659 & 0.001 \\
\hline $\begin{array}{l}\text { Residential } \\
\text { area }\end{array}$ & 0.207 & 0.892 & 0.337 & 0.798 & 0.463 & 0.708 & 0.955 & 0.414 \\
\hline Insurance & 1.866 & 0.116 & 0.617 & 0.651 & 1.428 & 0.225 & 2.884 & 0.023 \\
\hline
\end{tabular}

Table 2 Correlations between the main variables $(\mathrm{N}=320)$

\begin{tabular}{|lllll|}
\hline Variable & QOL & Bone pain & Skin pruritus & SDS \\
\hline QOL & 1 & & & \\
\hline Bone pain & $-0.509 * *$ & 1 & & \\
\hline Skin pruritus & $-0.517^{\star *}$ & $0.568^{* *}$ & 1 & 1 \\
\hline SDS & $-0.465^{\star *}$ & $0.450^{\star *}$ & $0.426^{* *}$ & 1 \\
\hline
\end{tabular}

Note: ${ }^{\star} P<0.01$

Table 3 The moderating effects of QOL (Part 1) 


\begin{tabular}{|lllllll|}
\hline Equation & $\begin{array}{l}\text { Dependent } \\
\text { variable }\end{array}$ & Independent variable & $\beta$ & SE & t & $\mathrm{P}$ \\
\hline Equation 1 & SDS & Bone pain & 0.485 & 0.054 & 8.984 & 0.000 \\
\hline Equation 2 & QOL & Bone pain & -0.407 & 0.039 & -10.548 & 0.000 \\
\hline Equation 3 & SDS & Bone pain & 0.310 & 0.060 & 5.190 & 0.000 \\
\hline & & QOL & -0.429 & 0.075 & -5.740 & 0.000 \\
\hline
\end{tabular}

Table 4 The moderating effects of QOL (Part 2)

\begin{tabular}{|lllllll|}
\hline Equation & $\begin{array}{l}\text { Dependent } \\
\text { variable }\end{array}$ & Independent variable & $\beta$ & SE & $\mathrm{t}$ & $\mathrm{P}$ \\
\hline Equation 1 & $\mathrm{SDS}$ & Skin pruritus & 0.375 & 0.045 & 8.404 & 0.000 \\
\hline Equation 2 & $\mathrm{QOL}$ & Skin pruritus & -0.337 & 0.031 & -10.757 & 0.000 \\
\hline Equation 3 & $\mathrm{SDS}$ & Skin pruritus & 0.223 & 0.050 & 4.510 & 0.000 \\
\hline & & $\mathrm{QOL}$ & -0.450 & 0.076 & -5.929 & 0.000 \\
\hline
\end{tabular}

Table 5 Results for total, indirect and direct effects of bone pain on SDS with QOL as a mediator

\begin{tabular}{|lccccc|}
\hline & SE & \multicolumn{2}{l}{ Bias-Corrected } & \multicolumn{2}{l|}{ Percentile } \\
\cline { 3 - 6 } & & $95 \% \mathrm{Cl}$ & & $95 \% \mathrm{Cl}$ & \\
\cline { 3 - 6 } & & Lower & Upper & Lower & Upper \\
\hline total effect & & & & & \\
\hline Bone pain-SDS & 0.436 & 0.279 & 0.585 & 0.276 & 0.581 \\
\hline indirect effect & & & & & \\
\hline Bone pain-QOL-SDS & 0.212 & 0.101 & 0.371 & 0.069 & 0.336 \\
direct effect & & & & & \\
\hline Bone pain-SDS & 0.224 & 0.053 & 0.43 & 0.061 & 0.438 \\
\hline
\end{tabular}

Table 6 Results for total, indirect and direct effects of skin pruritus on SDS with QOL as a mediator 


\begin{tabular}{|llllll|}
\hline & SE & \multicolumn{2}{l|}{ Bias-Corrected } & \multicolumn{2}{l|}{ Percentile } \\
\cline { 3 - 6 } & & $95 \% \mathrm{Cl}$ & & $95 \% \mathrm{Cl}$ & \\
\cline { 3 - 6 } & & Lower & Upper & Lower & Upper \\
\hline total effect & & & & & \\
\hline Skin pruritus-SDS & 0.61 & 0.435 & 0.795 & 0.433 & 0.792 \\
\hline indirect effect & & & & & \\
\hline Skin pruritus-QOL-SDS & 0.275 & 0.188 & 0.378 & 0.183 & 0.374 \\
\hline direct effect & & & & & \\
\hline Skin pruritus-SDS & 0.335 & 0.179 & 0.493 & 0.179 & 0.492 \\
\hline
\end{tabular}

Figures

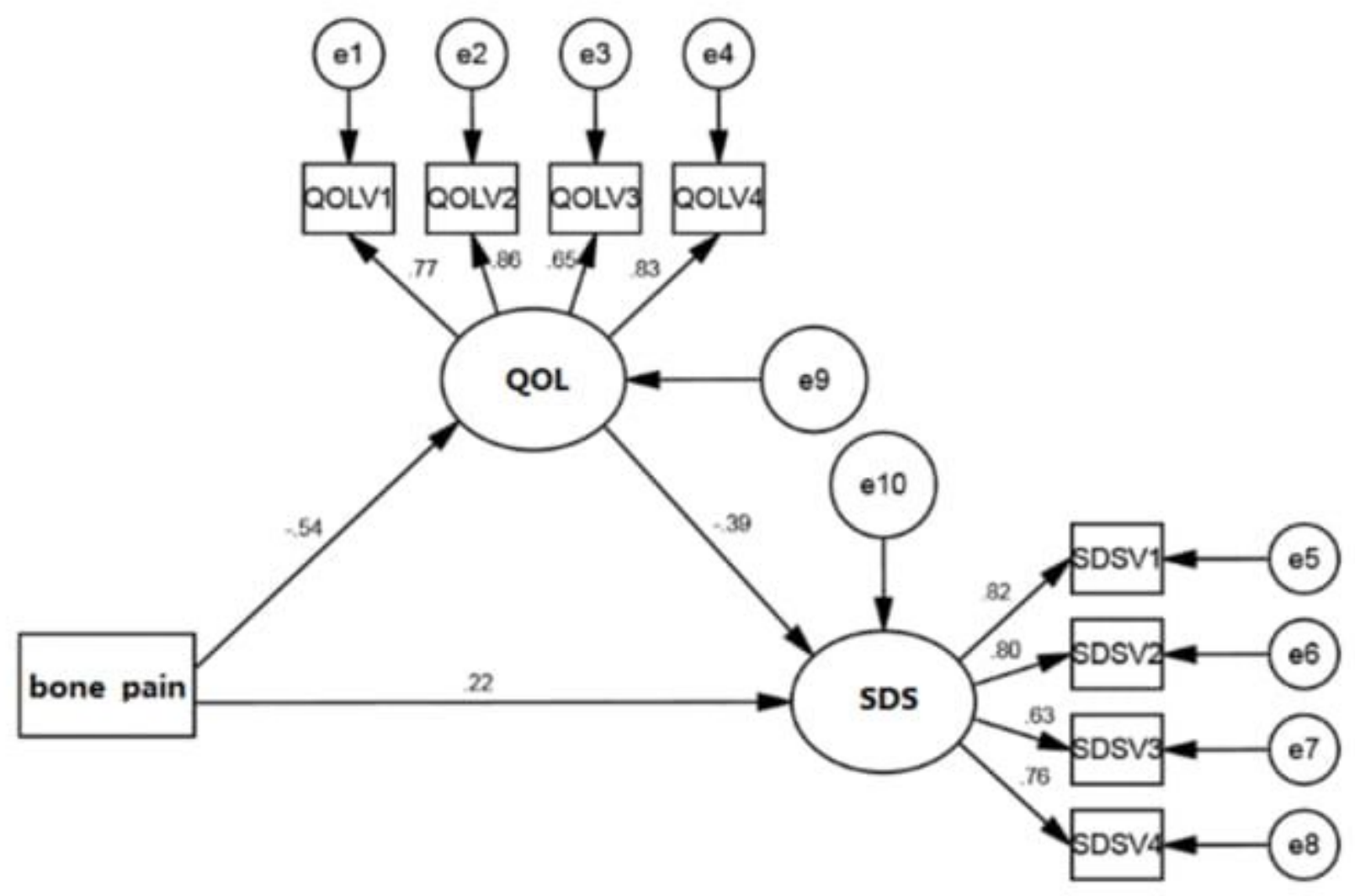

Figure 1 
Mediating effect of QOL on bone pain and SDS.

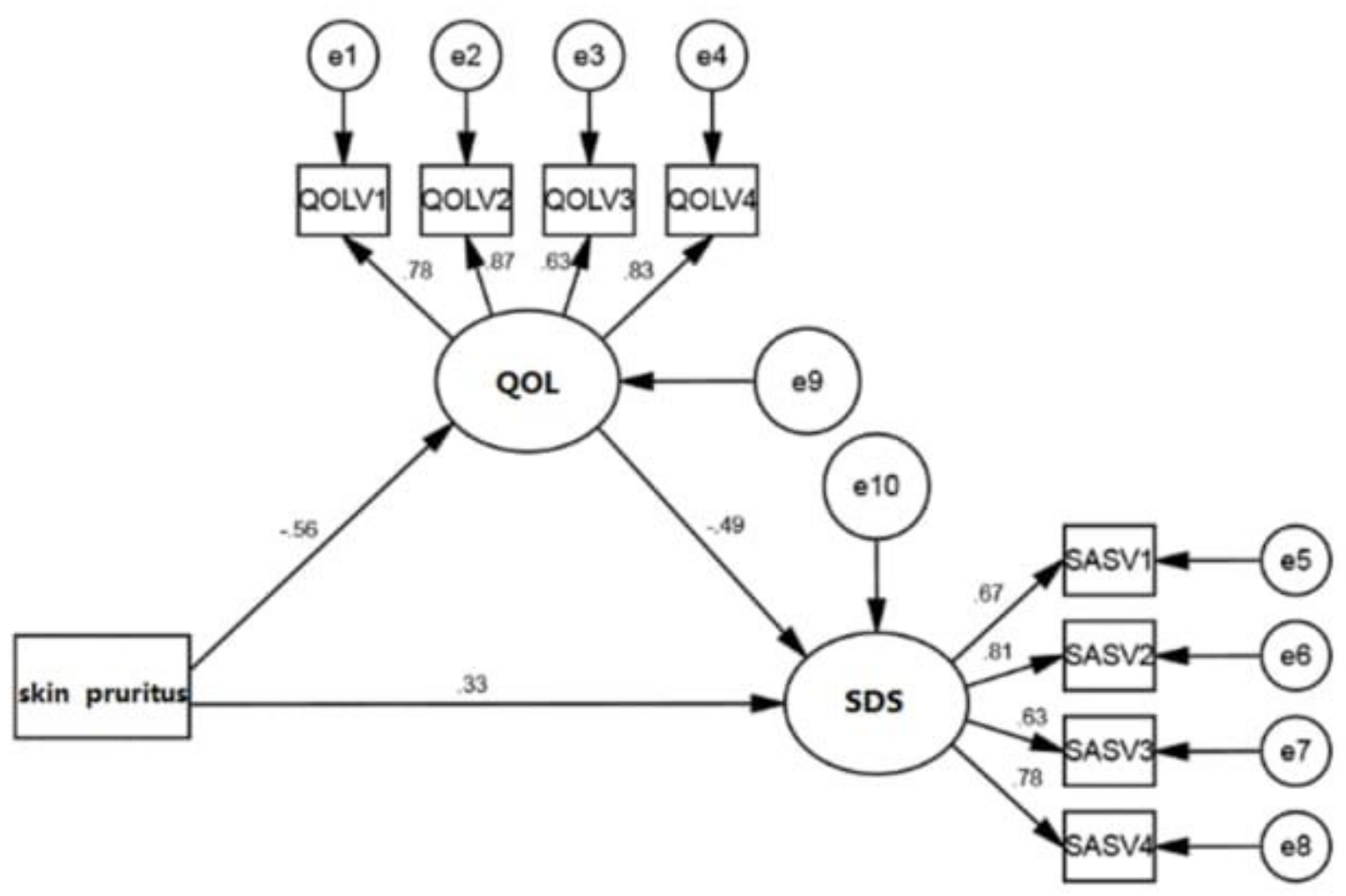

Figure 2

Mediating effect of QOL on skin pruritus and SDS.

\section{Supplementary Files}

This is a list of supplementary files associated with this preprint. Click to download.

- AdditionalTables.doc 